\title{
BUTTON BATTERY - AN UNWITNESSED CAUSE OF BATTERED NOSE
}

\author{
Shailesh R. Khode1 ${ }^{1}$, Nikesh Gosrani², Amitkumar Rathi' ${ }^{3}$, S.K.T. Jain ${ }^{4}$
}

\section{HOW TO CITE THIS ARTICLE:}

Shailesh R Khode, Nikesh Gosrani, Amitkumar Rathi, SKT Jain. "Button battery - an unwitnessed cause of battered nose". Journal of Evolution of Medical and Dental Sciences 2013; Vol2, Issue 48, December 02; Page: $9335-$ 9338.

ABSTRACT: The presence of foreign body in the nose is not uncommon condition. Various unusual foreign bodies in the nose have been reported in the literature in which button battery is one of them. It is capable of extensive tissue damage by chemical or thermal burns. We describe a case of an unwitnessed button battery in the nose of 3 years old female child resulted in septal perforation.

KEYWORDS: Nasal Foreign Body; Button Battery; Septal Perforation.

INTRODUCTION: Ear, nose and throat foreign bodies in children frequently present to both general practitioners and otorhinolaryngologist. Most of the foreign bodies are non hazardous like plastic objects, paper, cotton, cereals or foam. The dangerous foreign body like button battery has the potential to cause serious squeal if it is not removed immediately.

Button batteries are very commonly use energy source for various electronic devices. It is one of the common household items which are easily accessible and attractive to children to insert into nose, mouth and ear ${ }^{1}$. Just inserted batteries in nose can be removed in outpatient department. Those batteries have been left behind in nose for longer duration which is capable of extensive tissue damage, crusting and bleeding; their removal may be difficult so that general anesthesia will then be necessary.

These batteries generate local current leads to thermal as well as chemical burns resulting in necrosis, scarring, septal perforations and cosmetic deformity ${ }^{2}$. We present a case of unnoticed foreign body; button battery in nose which is complicated by septal perforation.

CASE REPORT: A 3 years old female presented to our outpatient department with history of fall followed by bleeding from the nose which was stop spontaneously. Anterior rhinoscopy examination revealed that edematous nasal mucosa with foul smelling mucoid discharge; blood clots and crusting. As narrated by parents, there was no history of foreign body insertion. Lateral view of Xray of nose and paranasal sinuses showed circular foreign body in the floor of the nostril (See figure 1).

Child was admitted, routine investigation done, started with injectable antibiotics and scheduled for general anesthesia next day. In Operation Theater, under Endoscopic guidance nasal secretion sucked out and after removal of crusting, heavily corroded button battery visualized which has been removed. On check endoscopy, a large perforation in cartilaginous part of septum was noticed (See figure 2). The surrounding edematous septal mucosa and lateral wall of nose were covered by blackish secretion and debris. The affected area is thoroughly irrigated with normal saline to avoid further damage by chemicals. The nasal cavity packed with medicated merocel which was removed after 48 hours. Child was discharged next day with prescribed saline nasal drops, antiinflammatory and antibiotic oral suspension. After 2 weeks follow up visit, anterior rhinoscopy revealed healthy nasal mucosa with septal perforation. 
DISCUSSION: The presence of foreign body in the nose is not uncommon condition between age of two to five years. They may be corrosive, hygrophillic or inert. Various unusual foreign bodies in the nose have been reported in the literature in which button battery is one of them. The ingestion of button battery in aerodigestive tract has known potentially fatal complications ${ }^{3}$. Different types of button batteries are invented such as silver, zinc, manganese, lithium, sulphur oxide, copper, brass, mercury and steel. Sodium hydroxide or potassium hydroxides are the contents of button battery which facilitate electrochemical reaction through the separator ${ }^{4,5}$.

We describe a case of 3 years old female child who inserted button battery in the nasal cavity. Moisture in the nasal cavity is responsible for corrosion of battery leads to release of its contents. Additionally battery can generate the current resulting in thermal burns and damages the nasal mucosa (an intense local tissue reaction and liquefactive necrosis) ${ }^{1,4}$.

Button battery has the potential to cause serious complications like mucosal necrosis, scarring followed by contraction, septal perforations and cosmetic deformity ${ }^{1,4}$. More the duration between insertion of battery and its removal, higher the risk of complications like septal perforation. Anode or cathode pole of battery orientation in the nasal cavity is also reported to be important related to damage (anode pole damages more than the cathode pole). The amount of nasal secretion and size of nose are also factor for the extent of damage ${ }^{1}$.

There are four potential mechanisms of button battery injury are nicely studied in animals which are as follows ${ }^{6}$.

1. Release of toxic substance (e.g. silver, zinc, mercury or lithium)

2. Local electrical burn- the electrical circuit completes when lodged in the aerodigestive tract and injury usually happens on the anode side of battery.

3. Electrolyte leakage from battery causing caustic injury.

4. Pressure necrosis caused by impacted foreign body.

Other contributing factors for extension and severity of injury by button battery are not well explained like duration of contact, site and size of battery6.

Most of the foreign bodies can be seen on anterior rhinoscopy examination but occasionally because of nasal secretion, crusting, mucosa edema or granulation, it is not visualized. Visualized foreign body is usually removed in outpatient department provided patient is cooperative. In our case, there was no history of foreign body insertion and the battery was not seen on examination. Xray nose lateral view required to visualize the foreign body. For extensive examination of nasal cavity general anesthesia was planned.

There are circumstances in which general anesthesia will be required such as uncooperative or very apprehensive patient, foreign body engulfed with granulation which is likely to be responsible for troublesome bleeding, posteriorly placed foreign body in nose with risk of slipping into the nasopharynx and lower airway, highly suspected foreign body which is not found on examination and needs extensive examination of nasal cavity?.

Patients with evidence of secondary infection, mucosal damage, congestion and granulation should received intravenous antibiotic and oral anti-inflammatory drugs. After removal of nasal pack, nasal cavity should be irrigated at regular interval. All patients require regular follow up visit for examination of nasal cavity and watch for any delayed sequelae. 
CONCLUSION: Button battery in nasal cavity is dangerous foreign body that can cause extensive tissue damage and long term complications. A thorough history, detail examination and radiological investigation help to diagnose the condition. We present this case to accentuate the endangerment of button battery insertion and to emphasize the gravity of early diagnosis and propitious management in such cases to avoid complications. Also invention of safety design of household items and toys, community awareness will definitely reduce the incidence of foreign body ingestion and/or insertion.

\section{REFERENCE:}

1. Loh WS, Leong JL, Tan HK. Hazardous foreign bodies: complications and management of button batteries in nose. Ann Otol Rhinol Laryngol. 2003; 112: 379-83.

2. Palmer O, Natarajan B, Johnstone A, Sheikh S. Button battery in the nose-an unusual foreign body. J Laryngol Otol. 1994; 108: 871-2.

3. Rajat Bhatia, Surinder K Singhal, Arjun Dass. Button cell causing septal perforation in a child. Clinical rhinology: An international journal. 2010; 3 (3): 161-3.

4. Kalan A, Tariq M. Foreign bodies in the nasal cavities: a comprehensive review of the aetiology, diagnostic pointers and therapeutic measures. Postgrad Med J. 2000; 76: 484-7.

5. Skinner DW, Chui P. The hazards of 'button -sized' batteries as foreign bodies in the nose and ear. J Laryngol Otol. 1986; 100: 1315-8.

6. Rajeev Jarugula, Tavey Dorofaeff. Oesophageal button batter injuries: thing again. Emergencies medicine Australasia. 2011; 23: 220-3.

7. Ransome J. Foreign bodies in the nose. In: Scott and Brown Otolaryngology. 1987, 5th ed., Butterworths, 276-9.

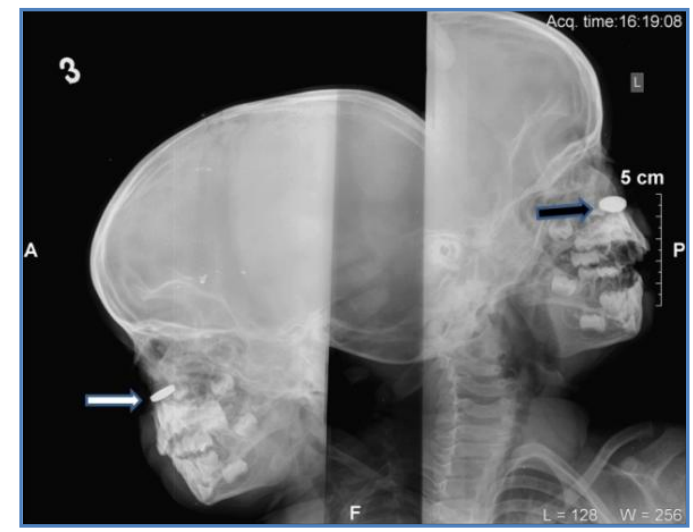

Fig. 1: Lateral view of X-ray of nose and paranasal sinuses showed circular foreign body in the floor of the nostril. 


\section{CASE REPORT}

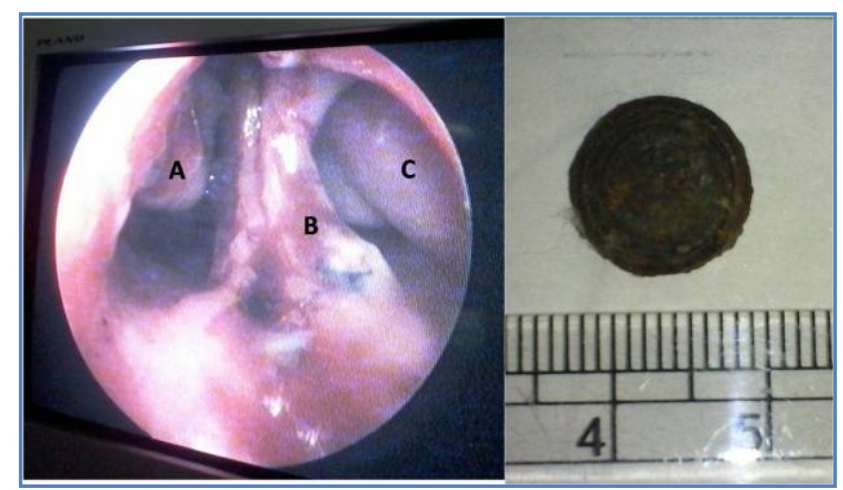

Fig. 2: Showed - A-Right side and C- Left side inferior turbinate and B- septal perforation. Another image showed corroded button battery.

\section{AUTHORS:}

1. Shailesh R. Khode

2. Nikesh Gosrani

3. Amitkumar Rathi

4. S.K.T. Jain

\section{PARTICULARS OF CONTRIBUTORS:}

1. Senior Lecturer, Department of ENT - Faculty of Medicine, MAHSA University College, Kuala Lumpur, Malaysia - 59100.

2. Resident, Department of ENT - Head \& Neck Surgery, Indira Gandhi Government Medical College \& Hospital, Nagpur, MS, India.

3. Resident, Department of ENT - Head \& Neck Surgery, Indira Gandhi Government Medical College \& Hospital, Nagpur, MS, India.
4. Professor and Head, Department of ENT Head \& Neck Surgery, Indira Gandhi Government Medical College \& Hospital, Nagpur, MS, India.

\section{NAME ADRRESS EMAIL ID OF THE CORRESPONDING AUTHOR:}

Dr. Shailesh R. Khode,

Senior Lecturer,

Department of ENT, Faculty of Medicine,

MAHSA University College,

Jalan Elmu off Jalan University Campus,

Kuala Lumpur, Malaysia - 59100.

Email - shaileshkhode@gmail.com

Date of Submission: 15/11/2013.

Date of Peer Review: 16/11/2013.

Date of Acceptance: 23/11/2013.

Date of Publishing: 26/11/2013 